\title{
Optimisation of the control laws for the SPHERE XAO system
}

C. Petit, T. Fusco, E. Fedrigo, J.-M. Conan, C. Kulcsár, et al.

C. Petit, T. Fusco, E. Fedrigo, J.-M. Conan, C. Kulcsár, H.-F. Raynaud, "Optimisation of the control laws for the SPHERE XAO system," Proc. SPIE 7015, Adaptive Optics Systems, 70151D (12 July 2008); doi:

$10.1117 / 12.790303$

EDIE Event: SPIE Astronomical Telescopes + Instrumentation, 2008, Marseille, France 


\title{
Optimisation of the control laws for the SPHERE XAO system
}

\author{
C. Petit*a, T. Fusco ${ }^{\text {a }}$, E. Fedrigo ${ }^{\text {b }}$, J.-M. Conan ${ }^{\text {a }}$, C. Kulcsár ${ }^{\mathrm{c}}$, H.-F. Raynaud ${ }^{\mathrm{c}}$ \\ ${ }^{\mathrm{a}}$ ONERA, BP 72, 92322 Châtillon, France \\ ${ }^{\mathrm{b}}$ Adaptive Optics Department, Telescope Systems Division, ESO - European Southern \\ Observatory, D-85748 Garching bei Munchen, \\ Germany \\ ${ }^{\mathrm{c}}$ Université Paris 13, Institut Galilée, L2TI, 93430, Villetaneuse, France
}

\begin{abstract}
The SPHERE (Spectro-Polarimetry High-contrast Exoplanet Research) instrument is an ESO project aiming at the direct detection of extra-solar planets. It should equip one of the four VLT 8-m telescopes in 2010. The heart of the SPHERE instrument is its eXtrem Adaptive Optics (XAO) SAXO (SPHERE AO for eXoplanet Observation) subsystem that should deal with a tight error budget. To fulfil SAXO challenging requirements a mixed control law has been designed. It includes both an optimized modal gain integrator to control the Deformable Mirror (DM) and a Linear Quadratic Gaussian (LQG) control law to manage the tip-tilt (TT) mirror and filter possible vibrations. A specific scheme has been developed to optimize the correction provided by the DM and the TT while minimizing the coupling between both control loops. Actuator saturation and wind-up effects management are described. We describe the overall control architecture and focus on these main issues. We present expectable performance and also consider the interactions of the main control loop with other subsystems.
\end{abstract}

Keywords: Adaptive Optics, Control

\section{INTRODUCTION}

The SPHERE system ${ }^{[1]}$ aims at detecting extremely faint sources (giant extra-solar planets) in the vicinity of bright stars. Such a challenging goal requires the use of a very-high-order performance Adaptive Optics (AO) system, a coronagraphic device to cancel out the flux coming from the star itself, and smart focal plane techniques to calibrate any coronagraph imperfections and residual uncorrected turbulent or static wavefronts. The detection limit for the SPHERE instrument is $10^{-6}$ (i.e. 15 magnitudes between star and planet) with a goal around $10^{-7}$. There is no simple link between the AO system performance and the final detectivity of the instrument. Nevertheless, the impact of AO on the final performance is related to the performance of the coronagraph. A better AO correction leads to better coronagraph extinction and therefore leads both to a reduction of the photon and flat-field noises and to a reduction of the static speckle. These reductions are important from the global system performance viewpoint and the optimization of the coronagraph rejection is a main goal of the SPHERE system. It of course requires the use and the optimization of an $\mathrm{XAO}$ system. The ultimate detection limit will be achieved through an extreme control of the system internal defects ${ }^{[2]}$ such as Non Common Path Aberrations (NCPAs), optical axis decentring, vibrations, coronagraph and imaging system imperfections and so on.

In this article, we focus on the main $\mathrm{AO}$ loop and in particular on the control architecture that has been designed to meet the requirements of SPHERE. The very high performance required having regards to the stabilization of the optical axis for the coronagraphic analysis in spite of the turbulence but also the overall system defects such as vibrations has lead to the definition of an original hybrid control law, separating the DM control from the tip-tilt mirror (ITTM) control. While the high order modes correction is ensured by the DM through a classic Optimized Modal Gain Integrator

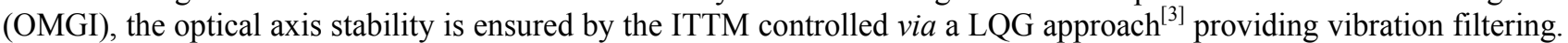
This hybrid control scheme relies on a decoupling procedure that forbids cross-talk. Though classic, the OMGI control has been however refined to manage the decoupling and ensure the highest performance in spite of inherent wind-up effects and possible DM saturation. The LQG approach, though particularly studied since a few years, has not been implemented yet on an operating system. It requires an extensive analysis and even if vibration filtering has already 
proved to be efficient thanks to this approach ${ }^{[4]}$, its implementation is still complex. Finally, ensuring the highest performance on a long time scale implies updating various parameters and matrices for the control.

This article is thus structured as follows. Sect. 2 presents SAXO main requirements from the control point of view and the consequences in terms of control architecture and chronogram. A global overview of the SAXO Real-Time control is then proposed in Sect. 3. In particular, the control strategy is presented and the separate control of DM and ITTM is justified. The decoupling scheme for this two-mirror parallel control is presented. Then Sect. 4 presents the DM control law based on OMGI. It lays the emphasis on the basis choice and building, and the anti-wind up procedure. Sect. 5 presents the ITTM control based on a LQG approach providing vibration filtering. The control law and the particular issue of ITTM control saturation handling are briefly described. Finally Sect. 6 presents performance of the overall SAXO and its control architecture based on end-to-end numerical simulation.

\section{SAXO REQUIREMENTS FROM THE CONTROL VIEWPOINTS AND CONSEQUENCES}

To meet the global requirements in terms of detection the SPHERE system is divided into four subsystems: the common path, including the SAXO XAO system with visible wavefront sensor and near infrared coronagraphic devices, which feeds the infrared dual-imaging spectrograph, IRDIS, the integral field spectrograph IFS and a visible dualimaging polarimeter ZIMPOL. The extreme AO system SAXO is the core of the SPHERE instrument and is essential for reaching the extremely high contrast requirements. Its design has been driven by the high level requirements.

\subsection{SAXO requirements and global architecture}

SAXO high level requirements have been extracted from the SPHERE Technical Specification and the SPHERE sub-system functional requirements. The main requirements concerning the AO loop are:

- Residual Tip-Tilt (TT), in normal conditions (seeing $=0.85 \operatorname{arcsec}$, average wind speed $=12.5 \mathrm{~m} / \mathrm{s}, \mathrm{L}_{0}=25 \mathrm{~m}$, Guide Star magnitude $<9$ in V, G0 star) is 3 mas rms (goal 1.5 mas). This leads to a dedicated control law, based on LQG, including prediction and vibration filtering.

- Turbulent residual wavefront variance on corrected modes in normal conditions is $60 \mathrm{~nm}$ rms. This leads to high bandwidth control law, an OMGI control on the high order modes (DM), to optimize the correction.

- $\operatorname{SR}(1.6 \mu \mathrm{m})$ is higher than $15 \%$ in poor (seeing $=1.1 \mathrm{arcsec}$, wind speed $=28 \mathrm{~m} / \mathrm{s}$ GS magnitude $<8$ ) or faint (normal conditions with GS magnitude < 12) conditions. It is also ensured by OMGI.

The main AO loop $(1.2 \mathrm{kHz})$ thus corrects for atmospheric and telescope defects. The main impact is the increase of detection signal to noise ratio through the reduction of the smooth PSF halo due to turbulence effects. The main AO loop also ensures pre-compensation for non common path aberrations and thus reduction of persistent speckle ${ }^{[5]}$. Additional control loops have been designed ( $\mathrm{se}^{[2,6]}$ for more details) such as the Differential TT (DTT) loop performing fine centering on coronagraph mask (correction of differential tip-tilt between VIS and IR channel), the pupil TT (PTT) loop providing pupil shift correction (telescope and instrument) based on the Shack-Hartmann visible WFS.

The AO system then consists of the following elements:

- a 40x40 visible Shack-Hartmann (VIS-WFS) measuring

$\bigcirc$ the atmospheric and common path phase perturbations,

$\circ$ the position of the telescope pupil,

- a high spatial $(41 \mathrm{x} 41$ actuators) and temporal $(1.2 \mathrm{kHz}$, goal $1.5 \mathrm{kHz})$ frequencies deformable mirror to correct for phase perturbations but the tip-tilt.

- a fast (1.2 Khz, goal $1.5 \mathrm{kHz})$ image TTM (ITTM) located in a pupil plane for image motion correction,

- a slow $(\sim 0.1 \mathrm{~Hz})$ pupil TTM (PTTM) close to the entrance focal plane to correct for pupil shifts,

- a slow (1Hz TBC) infra-red tip-tilt sensor (IR-WFS) on the scientific channel measuring the differential tip-tilt between the common and imaging paths, 
- a slow $(10 \mathrm{~Hz})$ differential image TTP (DTTP) located in a pupil plane (in the VIS-WFS path) to correct for differential tip-tilt between the imaging and VIS-WFS paths,

- a Real-Time Calculator (RTC).

These elements and the control loops managing them are presented in figure 1.

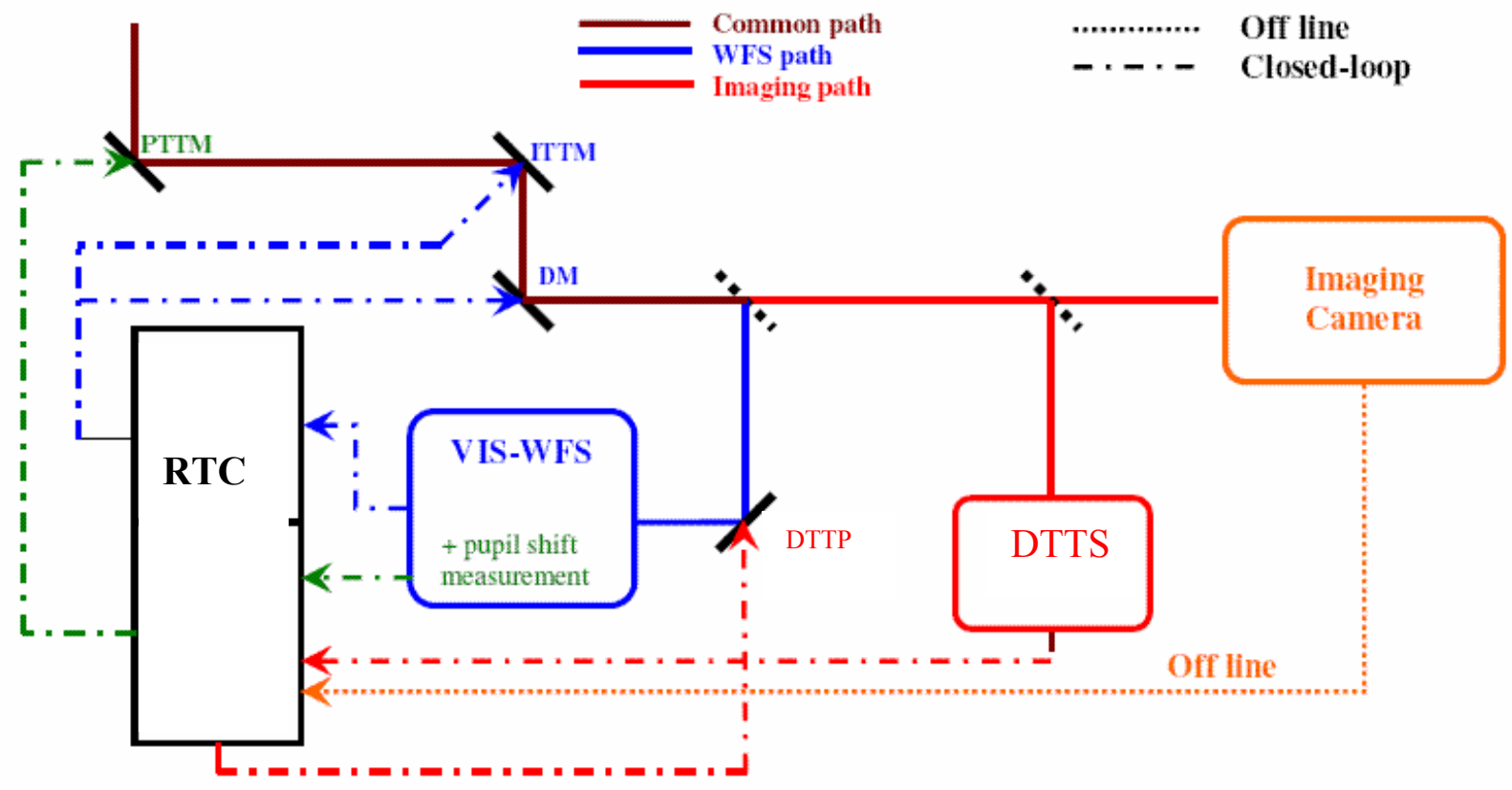

Figure 1: Schematic representation of SAXO.

In this article we only focus on the main AO loop, including the ITTM, DM, VIS-WFS and RTC. The SAXO various control loops are not all independent. Nevertheless, the PTT and the DTT loops are very low frequency $(<1 \mathrm{~Hz})$ control loops, so that we can assume decoupling of these control loops with the main AO control loop.

\subsection{SAXO RTC chronogram}

We consider here the main AO loop chronogram which represents the fastest and thus most demanding control loop. From the global AO performance point of view, the only two parameters to be set in this chronogram are the $\boldsymbol{C C D}$ integration time and the overall loop delay (defined between the first pixel read on the detector and the last voltage sent to the DM). The CCD integration time has been already defined in a contract with E2V and can not be adjusted during phase B. A minimum value of $833 \mu$ s with a goal value of $666 \mu$ s is expected (resp. 1.2 and $1.5 \mathrm{kHz}$ ) of course for bright stars, integration times going to $1000,1500,2000$ and $4000 \mu$ s should be considered for faint stars. It can be shown that from a turbulence point of view (in normal conditions), the temporal error is barely dependant from the sampling frequency between $1 \mathrm{KHz}$ and $1.5 \mathrm{KHz}$ but it is far more sensible to the AO loop overall delay. A delay of $1 \mathrm{~ms}$, with a $833 \mu \mathrm{s}$ goal, is a reasonable compromise in terms of performance and technical feasibility.

The delays which can be then optimized are (see figure 2) T1 $\Rightarrow \mathrm{T} 2$ (related to CCD controller), T7 $\Rightarrow \mathrm{T} 10$ (related to RTC) and T10 $\Rightarrow$ T11 (related to DM HVA). Various configurations may be chosen for a given global loop delay. A typical repartition could be the following:

- $745 \mu$ s of CCD read out (goal $666 \mu \mathrm{s})$,

- $55 \mu \mathrm{s}$ of CCD controller delay (goal $50 \mu \mathrm{s}$ ),

- $150 \mu \mathrm{s}$ of RTC latency (goal $100 \mu \mathrm{s}$ ),

- $50 \mu$ s of DM HVA (CODE) latency, 


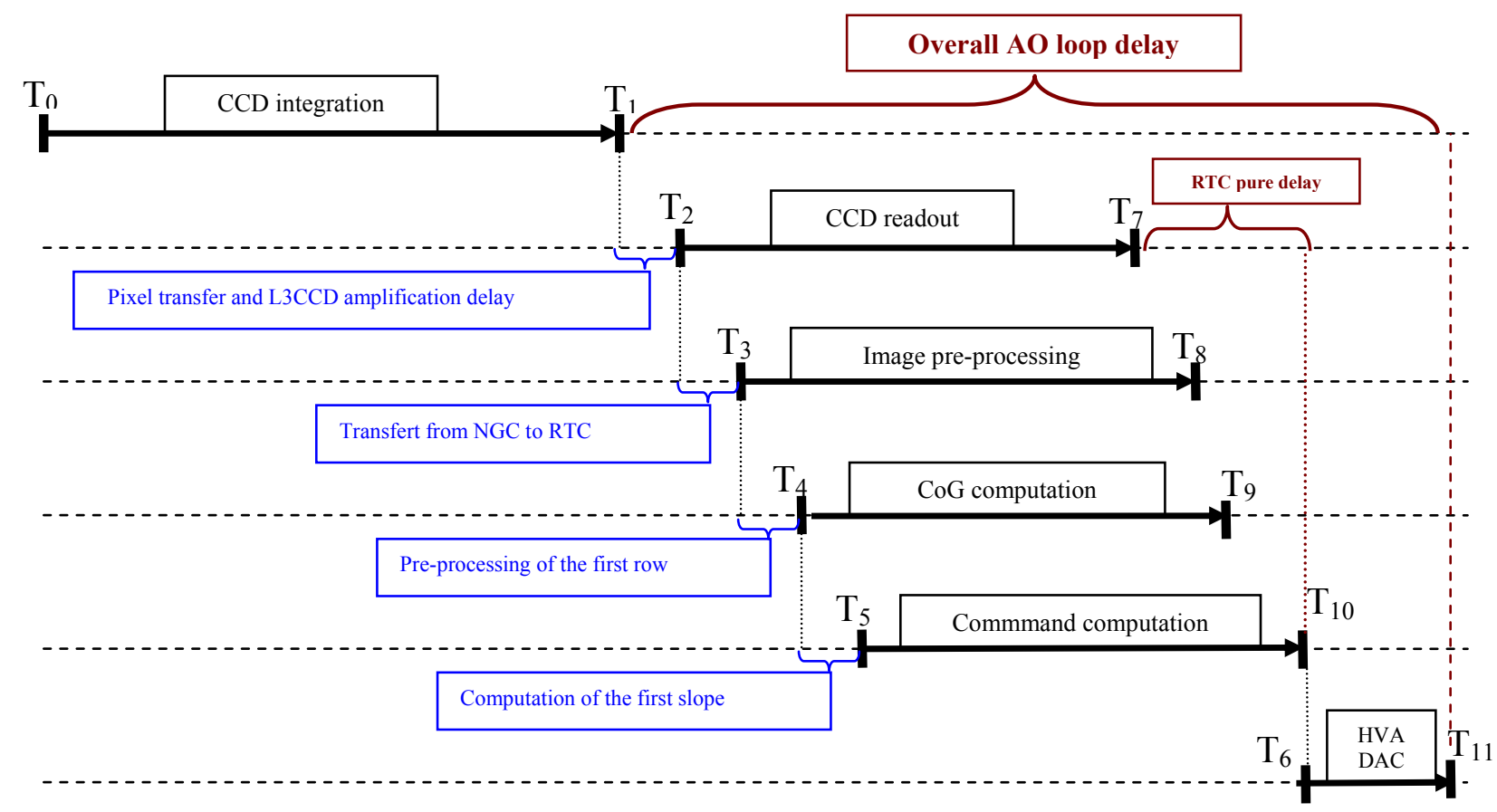

Figure 2: Main AO loop timing.

\subsection{Global overview of SAXO Real-Time Control}

\subsubsection{Control scheme overview: DM-Tip/tilt control separation strategy}

The SPHERE system design has been driven mainly by the concern to rely on proven technologies though pushed to their limit. The control strategy relies on the same policy. But due to the particularly high performance requirements of SPHERE, an original hybrid control scheme has been proposed. Thus, classic Optimized Modal Gain Integrator is an efficient, robust and well-known solution ${ }^{[7]}$. Other solutions, such as optimal control, through Linear Quadratic Gaussian approach, could have been considered ${ }^{[3]}$. Nevertheless, when applied to AO, LQG optimal control gain stems from the ability to predict and, through estimation of the turbulence on a high number of mode and regularization, its ability to reduce aliasing effects ${ }^{[8]}$. In the present case, the sampling frequency is greater than $1.2 \mathrm{KHz}$ reducing the gain brought by prediction and a spatial filter placed in the focal plan of the WFS automatically reduces the aliasing effect ${ }^{[9,10]}$. Thus the interest of a global LQG approach is reduced having regards to the complexity it induces. Still, two modes must be considered with a particular attention: the tip and tilt modes. These modes concentrate a large amount of turbulent energy, and, their drastic correction is a pre-requisite to coronographic imaging (residual TT should be less than 3 mas rms). The issue of tip-tilt modes correction becomes even more important in the presence of vibrations. It has been shown on the NAOS system that vibrations, due to windshake, cooling systems and so on, can greatly affect the performance of an $\mathrm{AO}$ systems ${ }^{[11]}$. It has been also underlined that these vibrations particularly affect the tip and tilt modes. Consequently, it appeared that a dedicated control law should be applied to the tip and tilt modes. Considering that LQG approach provides an accurate estimation and prediction of modes and in particular can handle vibration filtering in a global control law, this approach has been chosen to correct for the tip and tilt modes. Still, it is important to note first that the higher order modes would be corrected thanks to OMGI. This control law relies on a system modes approach as no estimation of the turbulence is made. And such a reconstruction would be particularly time consuming and computationally expensive. Then, tip and tilt modes are corrected by the tip and tilt mirror, even if in general this mirror also corrects more generally for the angle of arrival, which encompasses more than these two turbulent modes. Consequently, it has been chosen to dedicate the LQG control not to the tip and tilt turbulent modes correction but rather to the tip-tilt mirror control. This control architecture imposes nevertheless a strict decoupling between the controls of the two mirrors to avoid cross-talk that may result in instabilities. 


\subsubsection{Data processing}

The SAXO Real-Time processing is rather standard. It performs first an image pre-processing, consisting in dead pixels, background and flat-field correction. Slopes computation is performed thanks to Weighted Centre of Gravity (WCoG). The procedure, proposed by Nicolle et $a l^{[12]}$., ensures a dramatic reduction of the detector noise and it can be shown that WCOG reaches the lower bound in terms of noise propagation for a point like source. But WCOG has other interesting features even at high flux or in a photon noise regime when diffraction limited spots are considered (which is the case for SAXO because of the large number of sub-aperture and the presence of the spatial filter device). For instance, the WCOG algorithm dramatically attenuates the impact of secondary lobes of the diffraction pattern inducing in general errors in the CoG. It reduces indeed the weight of secondary lobes in the measurement process increasing the linearity and sensitivity. Finally slopes measurements are processed to ensure control laws decoupling before being sent respectively to the DM and TT control laws.

\subsubsection{Control laws decoupling}

The goal is to define a mixed control law, so that the DM is controlled thanks to an Optimized Modal Gain Integrator, while the ITTM is controlled thanks to LQG control, providing if necessary vibration filtering. It thus defines two control loops, one for each mirror. This control architecture is prone to coupling between the two control loops: although the control voltages are well separated between the ITTM and DM, just because the components are physically distinct, the slope measurement spaces encompassed by each mirror are not disjoint.

Separating the two control loops in two independent loops thus relies on separating the measurement spaces encompassed by each mirror. Let $E$ be the WFS measurement space, $E^{T T M}$ be the subspace of $E$ encompassed by the ITTM (image of the ITTM interaction matrix $D_{I T T M}$ ), and $E^{D M}$ the subspace of $E$ encompassed by the DM (image of the DM interaction matrix $D_{D M}$ ). $E^{T T M}$ is of dimension 2, and we assume that the rows of the ITTM interaction matrix noted $\left(S_{1}, S_{2}\right)$ represent an orthonormal basis of $E^{T T M}$, (a re-orthonormalisation is however possible if needed, through a Graham-Schmidt method). The intersection of $E^{D M}$ and $E^{T T M}$ is in general not empty. The goal is thus to define a subspace of $E^{D M}$ orthogonal to $E^{T T M}$ and to built the DM control law on this subspace.

Let $S$ be a turbulent slope measurement, and $D=\left(D_{I T T M}, D_{D M}\right)$ the general interaction matrix. The problem consists in finding the control voltages $v^{T}=\left(v_{I T T M}{ }^{T}, v_{D M}{ }^{T}\right)$ minimizing the residual slope measurement:

$$
\left\|S_{\text {res }}\right\|^{2}=\|S-D v\|^{2}=\left\|S-D_{\text {ITTM }} v_{\text {ITTM }}-D_{D M} v_{D M}\right\|^{2} .
$$

Defining for any slope measurement $S$, its projection $S_{/ /}$onto the subspace $E^{T T M}=\left(S_{1}, S_{2}\right)$, and its projection onto $E^{T T M}$ orthogonal subspace $S_{\perp}$, so that $S=S_{\perp}+S_{/ /}$with:

$$
S_{/ /}=\left\langle S \mid S_{1}\right\rangle S_{1}+\left\langle S \mid S_{2}\right\rangle S_{2}
$$

where $\langle\mid\rangle$ is a scalar product associated to the Euclidian norm \|\| .

We define the projection of $E^{D M}$ on $E^{T T M}$ computing $D_{\text {cross }}=\left(S_{1}, S_{2}\right)^{T} D_{D M}$. The kernel of this application defines the subspace of $E^{D M}$ orthogonal to $E^{T T M}$. Taking into account orthogonality we deduce:

$$
\left\|S_{\text {res }}\right\|^{2}=\left\|S_{/ /}-D_{\text {ITTM }} v_{\text {ITTM }}-\left(D_{D M} v_{D M}\right)_{/ /}\right\|^{2}+\left\|S_{\perp}-\left(D_{D M} v_{D M}\right)_{\perp}\right\|^{2} .
$$

Quadratic error term $\left\|S_{\perp}-\left(D_{D M} v_{D M}\right)_{\perp}\right\|^{2}$ should then be minimized onto this subspace to ensure $\left(D_{D M} v_{D M}\right)_{/ /}=0$ while $\left\|S_{/ /}-D_{\text {ITTM }} v_{\text {ITTM }}\right\|^{2}$ is minimized independently. During this operation 2 degrees of freedom of the DM are lost, but this is the sine qua non condition to ensure $\left(D_{D M} v_{D M}\right)_{/ /}=0$ and the decoupling. 


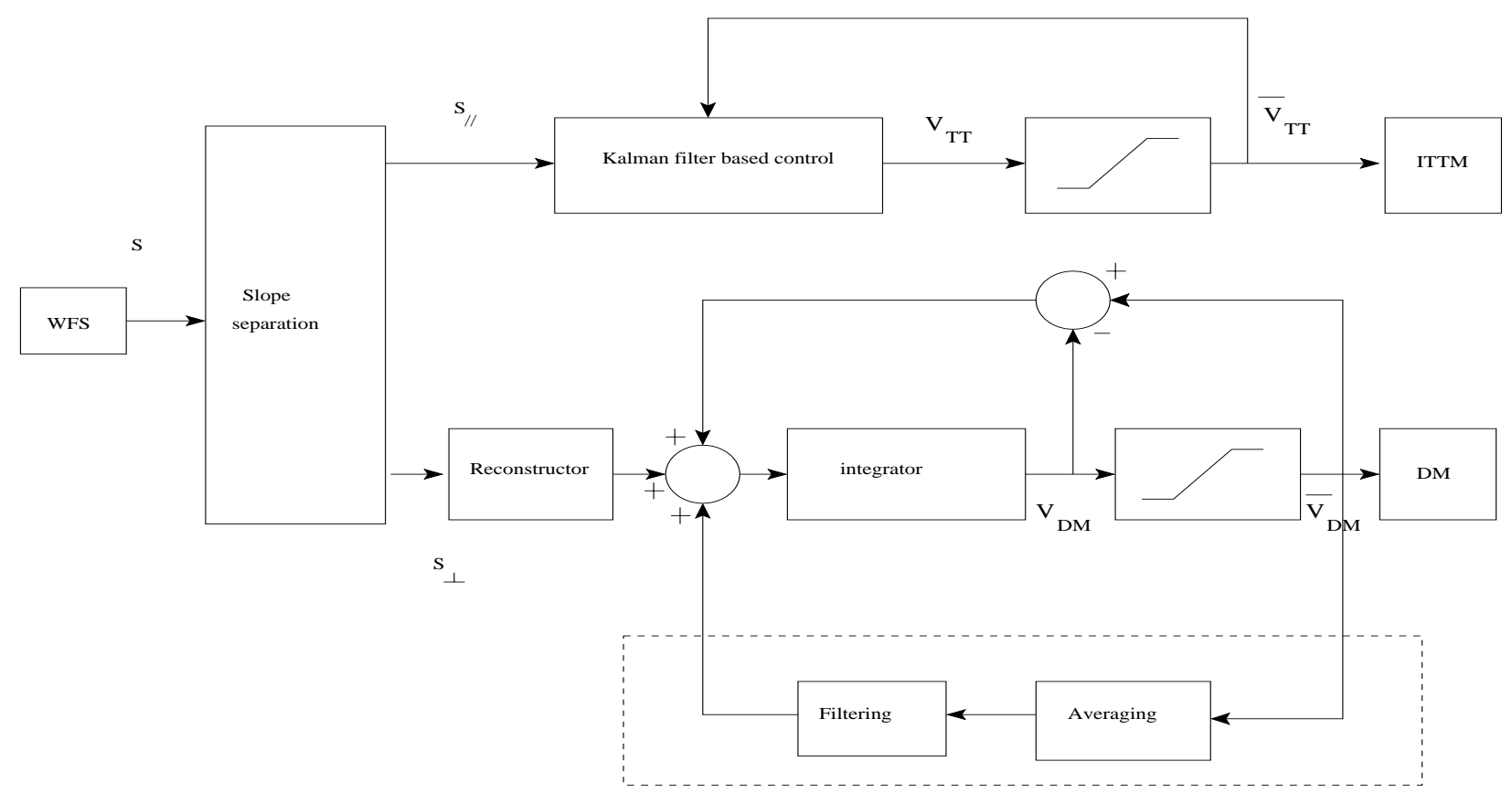

Figure 3: Overall AO control simplified representation.

Numerical analysis of this decoupling procedure on an end-to-end simulation of the full SAXO system has shown a very efficient decoupling between DM and TT channels. Decoupling was estimated computing the quantity $\left\langle\left\|\left(D_{D M} v_{D M}\right)_{/ /}\right\|^{2}\right\rangle$ and proved to be many orders of magnitude lower than classical solutions (mean slope solution for instance). Robustness analysis is being performed for SPHERE Final Design Review.

Note that it is not strictly equivalent to minimizing (1) as a constraint is imposed and the degrees of freedom of the DM are reduced. We only assume that the degrees of freedom lost are entirely compensated for by the ITTM and that it does not reduce the ability of the mirror to correct for the other modes. Note also that the 2 modes of $E^{D M}$ belonging also to $E^{T T M}$ are thus not controlled and evolve in open-loop, meaning that these 2 modes should be included in an anti-windup procedure to avoid amplification.

This procedure allows decoupling between the ITTM and DM control loops. The ITTM control is ensured by a LQG control. The DM control is ensured by an OMGI on the kernel of $D_{\text {cross }}$. This procedure is very similar to the classic procedure applied when controlling a DM and a tip-tilt mirror thanks to global command matrix and integrator, except that usually the mean slope measurement is used in place of $\left(S_{1}, S_{2}\right)$, leading to a poorer decoupling. We denote in the following by turbulent ITTM slope modes the projection of the turbulence measurement onto the $\left(S_{I}, S_{2}\right)$ modes.

\section{DM CONTROL}

The DM control is based on a classic optimized modal gain integrator. We first discuss the choice of modal basis and truncation issues then describe the procedure for saturation handling.

\subsection{OMGI overview}

Various modal basis can be used for the integrator based DM control and its optimization. Basis derived only from the turbulence characteristics (Zernike basis) do not seem suitable as they are computed without respect to the whole AO system properties. Usually, modal basis are on the contrary defined only with respect to the whole system. The simplest solution is then the eigen modes of the system, computed from a Singular Value Decomposition (SVD) of the interaction 
matrix. They provide a good analysis of the modes the system is able to measure and correct. Nevertheless, these modes are totally disconnected from the turbulence characteristics and do not account, for instance, for the particular distribution of turbulent energy according to spatial frequencies and noise. An analysis of the eigen modes of SAXO shows (Fig. 4.) indeed that while the badly seen ones (low eigen values) are related to waffle modes, the modes associated to high eigen values are more related to Fourier modes, due to the high number of actuators. It also appears that optical modes like coma or astigmatism can be found among modes with low eigen values (see Fig. 4). Classic computation of the command matrix, through SVD and truncation of the modes related to low eigen values, will thus be more damageable to the overall loop performance.

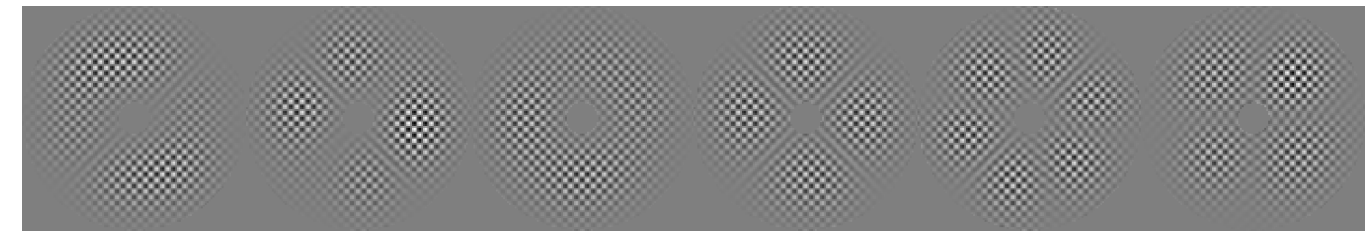

12

3

4

5

6

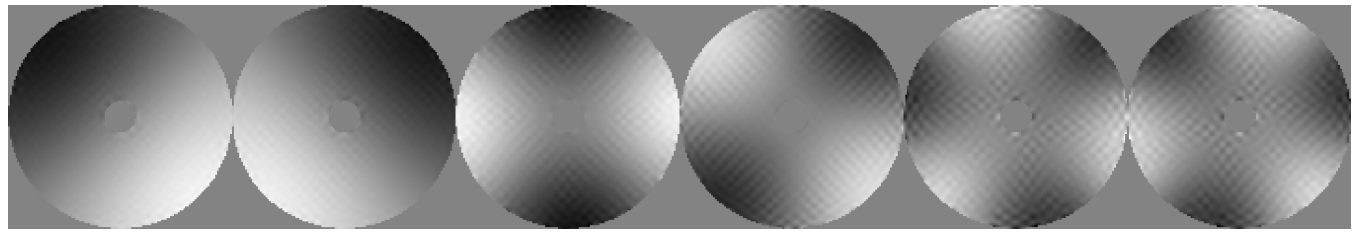

68

69

105

106

140

141

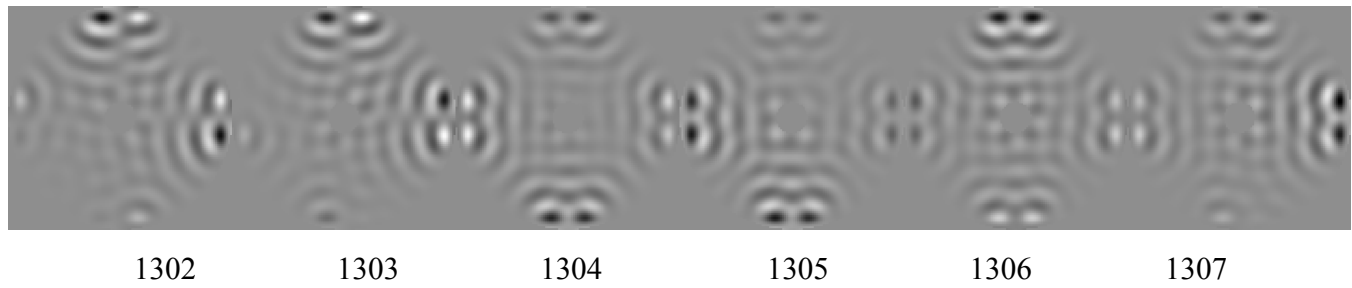

Figure 4: SAXO eigen modes, ordered by increasing eigen values (mode number below). The first ones (top) are related to waffle modes, the last (bottom) to Fourier modes. But among the first eigen modes (middle) also exists highly energetic turbulent modes

(very close to coma, astigmatism ...).

An alternative has thus been proposed, considering the projection of the turbulent wavefront space onto the space defined by the DM influence functions. A Karhunen-Loeve basis (KL) can be computed on this space ${ }^{[13]}$. In the present case, this KL basis must also been computed accounting for the decoupling, that means the KL are computed on the kernel of $D_{\text {cross }}$. The KL modes are naturally ordered according to the propagated turbulent energy (with a $-11 / 6$ asymptote), but they are also roughly well ordered according to the propagated measurement noise (with a -1 asymptote). This result is not quite surprising, as the KL modes are, in a first approximation, ordered according to spatial frequencies, the first ones being well measured by the SH-WFS and very similar to Zernike modes (see Fig. 5). This property is particularly favourable when performing truncation. Truncation is actually performed directly on the KL basis prior to the interaction matrix computation, suppressing the last high frequency related modes. This result is confirmed by end-to-end simulation of the full SAXO system and computing either a standard eigen mode basis or the KL basis. Then the overall residual error of the system is evaluated according to the truncation level, and after full modal gain optimisation on each basis, for various flux (Fig. 6). The KL mode basis provides an equivalent or better performance with less sensitivity of the performance to truncation. A truncation of 100 modes provides a sufficient performance in most conditions.

In practice, the interaction matrix is expressed on the truncated KL basis; the command matrix is then computed naturally thanks to SVD. The integrator modal gain optimization is performed on the KL basis and is based on the standard procedure developed by Dessenne ${ }^{[14]}$. 

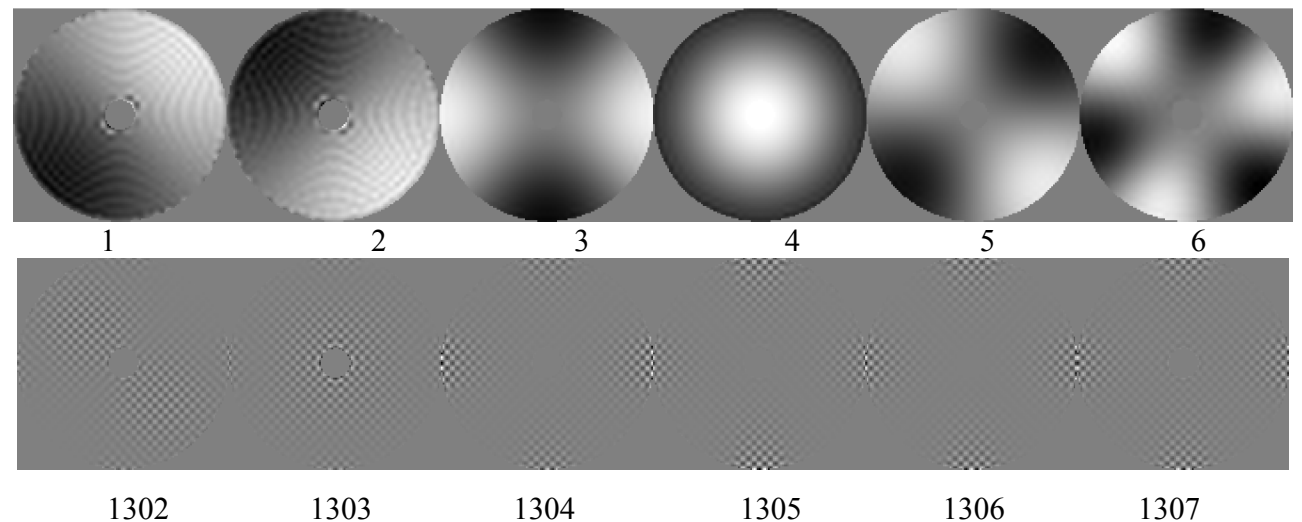

Figure 5: SAXO KL modes, ordered by increasing mode number, which is also by decreasing turbulent energy.
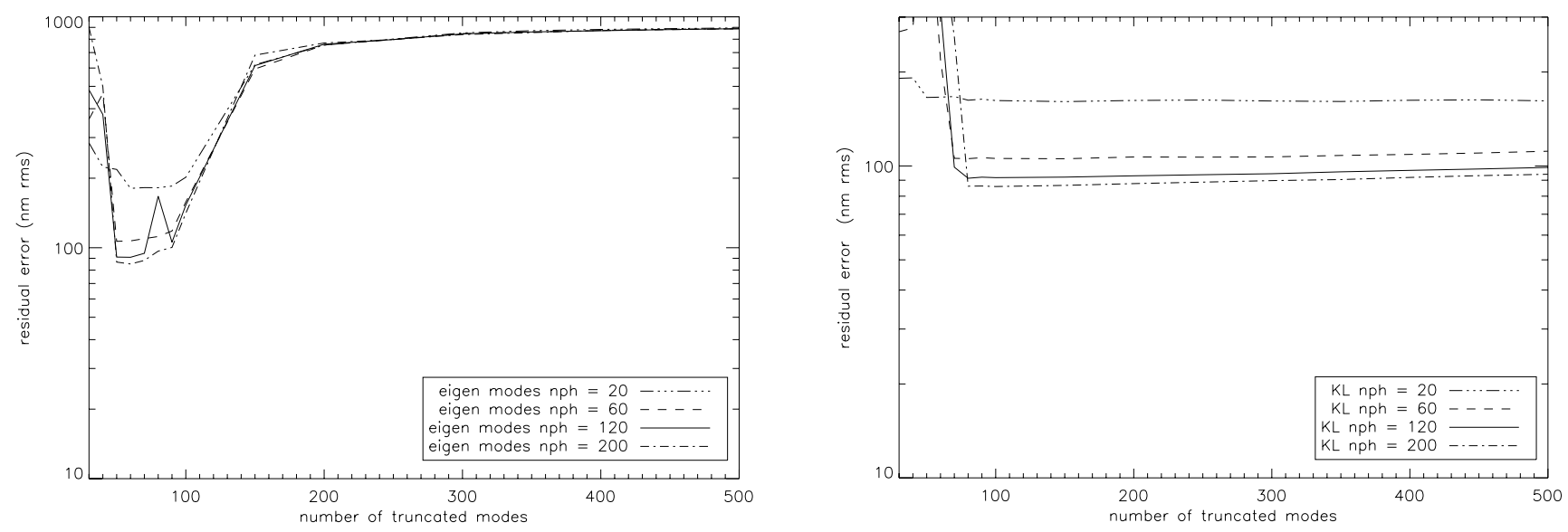

Figure 6: Impact of basis truncation respectively for the eigen mode basis (left) or the KL basis (right) for various flux (20, 60, 120 and 200 photons/frame/sub-aperture) in terms of overall residual error ( $\mathrm{nm} \mathrm{rms).}$

\subsection{Saturation Handling}

SAXO DM has of course a finite stroke, specified during system design, taking into account various turbulent conditions to face. Still, unexpected bad turbulent conditions may appear, leading to DM saturation. Protection of the DM is ensured by DM commands clipping. But clipping is a non-linear operation that may lead to project the computed DM voltages onto "fancy" unseen modes, thus evolving in open-loop. This participates to the well known wind-up effect, characteristic from integral control law. It is thus necessary to monitor the evolution of these modes.

The unseen modes of the DM are:

- The 2 voltage modes generating slope measurements collinear to the ITTM slope measurements $\left(S_{1}, S_{2}\right)$;

- The filtered modes of the KL basis.

It should be then necessary to project each clipped voltages onto these modes, but the computation cost would be deterring. The solution proposed relies on a garbage collector (box in dashed line on Fig.2) proposed by E. Fedrigo, averaging the clipped DM voltages over a given time, applying a filtering of the average value (projection onto the problematic modes), and providing to the integrator input an error term at a $1 \mathrm{~Hz}$ frequency (goal $5 \mathrm{~Hz}$ ). 


\section{TIP-TILT CONTROL}

The residual tip tilt motion is a critical item for the SPHERE performance. The extremely challenging value required for coronagraph optimal performance has lead us to design a particular control for the TT based on LQG control, providing estimation, prediction and correction of vibrations in addition to turbulent modes.

\subsection{ITT control scheme overview}

The ITT control is performed thanks to a LQG control, including estimation by a Kalman filter and correction by direct least-square projection onto the TT mirror assuming it has a perfect dynamic ${ }^{[3]}$. The input of the control loop is the projection of the SH-WFS onto the ITT turbulent slopes modes $\left(S_{1}, S_{2}\right)$. The output is control voltages to be sent to the TT mirror, if necessary clipped to avoid saturation. Offload toward the telescope is also performed to manage global drift of the TT.

The LQG control proposed is based on the solution described in $^{[3]}$, but restricted to the sole turbulent and vibration $\left(S_{1}, S_{2}\right)$ modes. Note that in this case, the LQG control does not provide optimal control with respect to the turbulent residual phase variance, but linear optimal control with respect to the turbulent $\left(S_{1}, S_{2}\right)$ modes residual variance. Still, the optimal estimation and prediction of these modes relies on the same procedure as the one described in ${ }^{[3]}$. It implies in particular priors on the system (models of the TT mirror or influence functions, of the WFS measurement and noise), and the turbulent modes to estimate (state model). In the particular SAXO case, the system priors are very simple due to the modes considered: the $\left(S_{1}, S_{2}\right)$ directly corresponds to the TT mirror modes and their measurement by the WFS leading to identity matrices for ITTM and WFS. State model of these 2 modes are also easily derived from a global turbulence model, based on Kolmogorov statistics and an Auto-Regressive (AR) model. In the SAXO case, second or third AR models have been considered to provide higher performance with better prediction for a limited complexity.

In addition to the turbulent modes, vibrations on these modes can be estimated and corrected. Up to 3 (goal 10) vibrations on each mode can be thus filtered thanks to a procedure detailed in ${ }^{[4]}$. Second order ARs are used to model each vibration. A particular identification procedure ${ }^{[15]}$ has been designed to provide on-line identification of the vibrations parameters from closed-loop measurements and update the LQG control matrices.

\begin{tabular}{|l|l|l|l|}
\hline & Vibration 1 & Vibration 2 & Vibration 3 \\
\hline Central frequency (Hz) & 50 & 120 & 310 \\
\hline FWHM (Hz) & 0,5 & 0,8 & 2 \\
\hline Cumulated energy (in mas ${ }^{2}$ (open-loop) & 2.8 & 3.92 & 1.6 \\
\hline Residual energy after filtering ( in mas $\left.{ }^{2}\right)$ & $<0.02$ & 0.06 & 0.2 \\
\hline
\end{tabular}

Table 1: Characteristics of the three vibrations introduced.

As an illustration, end-to-end numerical simulation of the SAXO system in presence of vibrations has been performed. 3 vibrations have been added on one of the $\left(S_{1}, S_{2}\right)$ modes, in addition to the turbulence, their characteristics are described in Table 1. DM control is performed as described in Sect. 4, and LQG control is performed on the $\left(S_{1}, S_{2}\right)$ modes with or without vibration filtering. Figure 7 shows the impact of vibration filtering compared to the input signal in terms of PSD and compared to no filtering, in terms of cumulated PSD. it proves a drastic filtering of the vibration and improvement of the global performance, as the residual image motion initially of 2.1 mas rms goes up to 4.32 with vibrations (not filtered) and falls down back to 2,28 when filtering is applied. It can be seen on the cumulated DSP an increase of residual energy at low frequencies related to a loss of low frequency turbulence rejection: vibration filtering implies an increase of rejection at the vibration frequencies that must be redeemed by a loss of rejection (Bode theorem). Still the performance improvement directly impacts the coronagraphic performance of the SAXO (increase of coronagraphic rejection).

As a conclusion LQG control allows to reduce both the residual tip tilt (due to turbulence effects) by more than $25 \%$ (this value depends on the turbulence characteristics) due to optimal estimation and prediction and also to filter efficiently vibrations for a limited complexity. Robustness and identification issues are currently under study. 

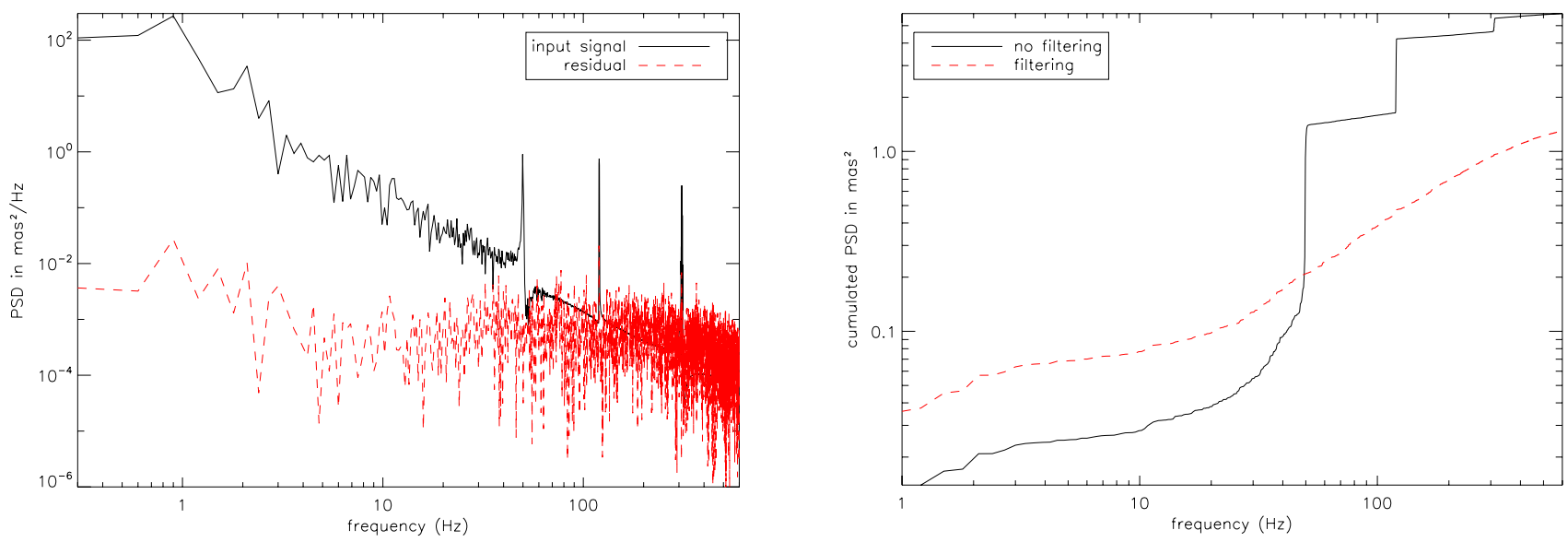

Figure 7: left, input and residual PSD of the $S_{2}$ mode on which the vibrations are introduced with vibration filtering. Right: cumulated PSD of residual $S_{2}$ mode with or without filtering.

\subsection{ITT mirror saturation handling}

Considering the ITTM control loop, due to the ITTM course and the offload procedure, saturation should seldom occur. Still, saturation must be handled if necessary and considering the LQG control applied a specific scheme must be considered. A rather straightforward solution is proposed, that allows accounting for the saturation in the estimation process, thus reducing the risks of instability and the duration of saturation for a limited complexity. Nevertheless, this solution does not prevent from saturation and leads to a loss of performance when saturation occurs.

The solution proposed consists in taking into account in the Kalman filter the control voltages really applied to the DM, which means including the voltages clipping. This solution only consists in avoiding model errors due to clipping in the estimator. The Kalman filter then still provides the optimal estimate of the turbulent ITTM slope modes. But, correction is no more optimal, as a simple mean-square projection is performed, not accounting for the saturation problem. Then clipping occurs. This solution has proved ${ }^{16]}$ of course a better performance than no accounting of clipping. This leads to the control architecture proposed in Fig. 2. A more efficient solution could be derived as in ${ }^{[16]}$ through penalization (the quadratic criterion is added to reduce the control energy and thus reduce the saturation risk) or projection under constraint. But once more, saturation should hardly occur due to offloading.

\section{GLOBAL PERFORMANCE SIMULATION}

Results presented below have been obtained thanks to an end-to-end numerical simulator of the full SAXO system. This simulator has been cross-checked with the analytical code used during SAXO phase A studies. This simulator is based on the following modules: a multi-layer atmosphere simulator, a filtered Shack-Hartmann Wave-Front Sensor (WFS) module, a Tip-Tilt and Deformable Mirror (DM) module, control laws module, imaging module with coronagraphic imaging. Simulation and correction of vibrations can be introduced. Figure 8 shows the overall AO performance with two different control laws and for different flux ranking from 1 to 10000 photons/sub-aperture/frame (typically representing a 9 Guide Star magnitude). Both control laws are based on ITTM slope modes decoupling, and use an OMGI for higher order modes correction defined on the KL modal basis. The number of filtered modes has been set to 100 , which proves to be a good average value, but could be nonetheless optimized further for each flux. Then first control law uses a classic optimized modal gain integrator for ITTM slope modes while second control law uses a LQG control for ITTM modes, with a second order AR model for turbulent $\left(S_{1}, S_{2}\right)$ modes.

Figure 9 shows coronagraphic images obtained for various flux with the full control law including decoupling, OMGI on the higher modes and LQG control for the ITTM. Results for the two control laws are quit similar, in particular concerning the residual error (Fig. 8 left), and for high flux. A slight gain of performance in final residual error is observed at low flux for the LQG plus OMGI control, while it provides a systematic gain in residual image motion, up to 
1 mas at very low flux (Fig. 8 right). Further performance improvement is of course expected when vibrations are corrected for as shown in Sect 4.
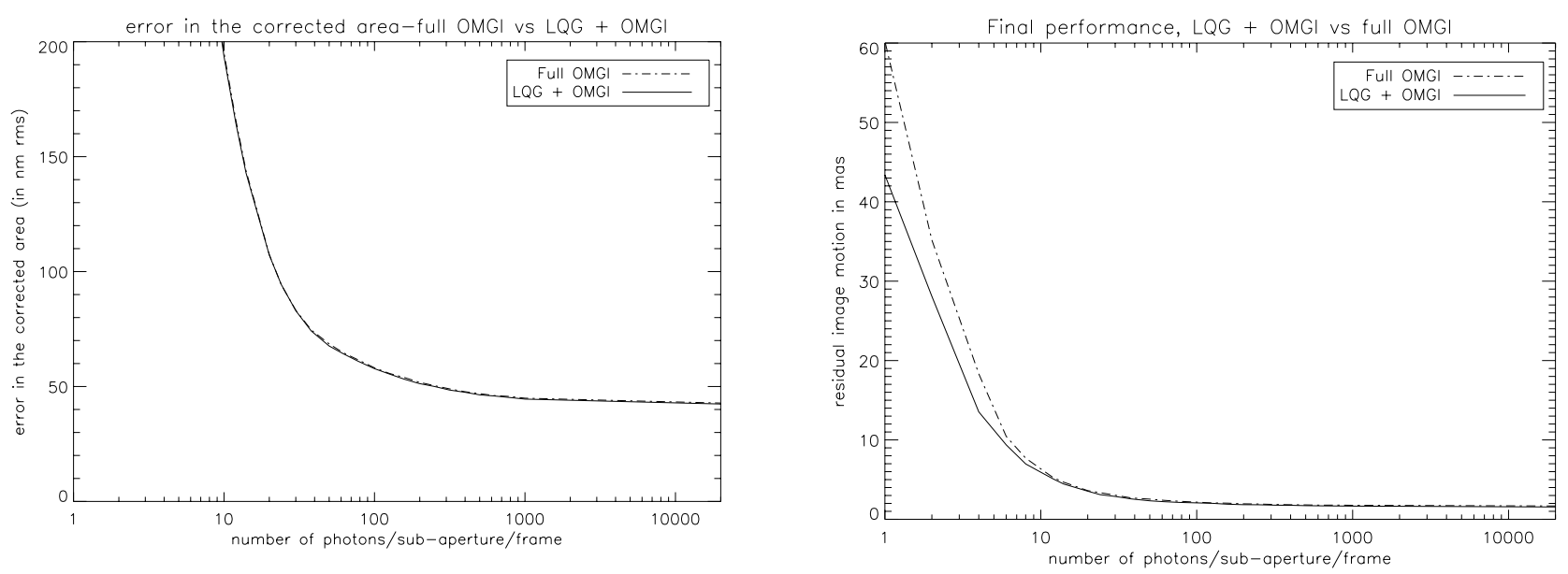

Figure 8: left SAXO final performance in residual error (in $\mathrm{nm} \mathrm{rms)}$ ) according to flux. Right: SAXO final performance in residual image motion (in mas rms) according to flux.

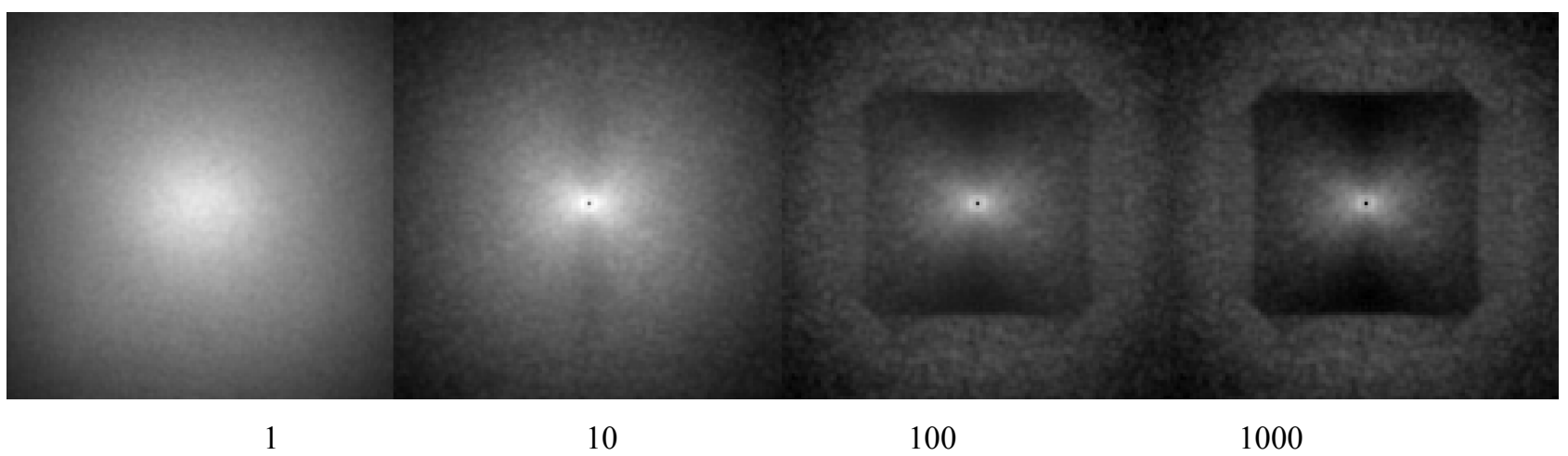

Figure 9: Coronagraphic PSFs for various flux in photons/sub_aperture/frame.

\section{CONCLUSION}

We have proposed an overview of the SAXO control architecture and its main particularities. SAXO high level requirements lead to a particular control design, based on a hybrid control law. While the DM is controlled through classic OMGI on a KL basis, the TT mirror is controlled thanks to LQG control, providing both optimal estimation/prediction, and vibration filtering. The original architecture imposes a strict decoupling procedure to avoid cross-talk, and a more complex management of the control laws and their interaction with the SPHERE system, such as offloading, matrices and parameters updates and so on. Still, this control architecture provides very good performance well inside the SAXO requirements. The performance of the LQG control will of course depend on the number and nature of the vibrations to be filtered. Identification procedures to update the various control matrices, particularly for vibrations tracking, are already being tested ${ }^{[15]}$. Still, experimental data from telescope are required to precisely define the LQG control and the identification process and their final performance. This issue should be tackled during the Final 
Design Review, along the global control architecture refining and robustness analysis, including in particular the decoupling procedure.

\section{ACKNOWLEDGEMENT}

The authors thanks all the SPHERE team for fruitful discussions.

\section{REFERENCES}

[1] J.-L. Beuzit, D. Mouillet, C. Moutou, K. Dohlen, P. Puget, T. Fusco, and A. Boccaletti, "A planet finder instrument for the VLT," in Proceedings of IAU Colloquium 200, Direct Imaging of Exoplanets: Science \& Techniques, Cambridge University Press, pp. 317-323, (2005)

[2] T. Fusco, G. Rousset, J.-F. Sauvage, C. Petit, J.-L. Beuzit, K. Dohlen, D. Mouillet, J. Charton, M. Nicolle, M. Kasper, P. Baudoz, and P. Puget, "High-order adaptive optics requirements for direct detection of extrasolar planets: Application to the SPHERE instrument, " Opt. Express 14, 7515-7534 (2006)

[3] C. Petit, J.-M. Conan, C. Kulcsár, H.-F. Raynaud, T. Fusco, J. Montri and D. Rabaud, "Optimal Control for Multiconjugate Adaptive Optics, ” Comptes Rendus de l’Académie des Science Physique 6, 1059-1069, (2005)

[4] C. Petit, J.-M. Conan, C. Kulcsár, H.-F. Raynaud, and T. Fusco, "First laboratory validation of vibration filtering with LQG control law for Adaptive Optics, "Opt. Express 16, 87-97 (2008)

[5] J.-F. Sauvage, T. Fusco, G. Rousset, C. Petit, A. Blanc, B. Neichel, and J.-L. Beuzit, "Fine calibration and precompensation of non-common path aberrations for high performance AO system," in Astronomical Adaptive Optics Systems and Applications II, R. K. Tyson and M. Lloyd-Hart, eds., Proc. SPIE 5903, 88-95 (2005).

[6] C. Petit, T. Fusco, J. Charton, D. Mouillet, P. Rabou, G. Rousset, P.Gigan, M. Kasper, E. Fedrigo, N. Hubin, P. Feautrier, J.-L. Beuzit, P. Puget, "The SPHERE XAO System: Design and Performance", this SPIE conference, paper 7015-65.

[7] E. Gendron and P. Léna, "Astronomical adaptive optics I. Modal control optimization," Astron. Astrophys. 291, 337-347 (1994).

[8] B. Le Roux, J.-M. Conan, C. Kulcsár, H.-F. Raynaud, L. Mugnier and T. Fusco, "Optimal control law for classical and multiconjugate adaptive optics, " J. Opt. Soc. Am. A 21, 1261-1276, (2004).

[9] L. A. Poyneer and B. Macintosh, "Spatially filtered wave-front sensor for high-order adaptive optics," J. Opt. Soc. Am. A 21, 810-819 (2004).

[10] T. Fusco, M. Nicolle, G. Rousset, V. Michau, J.-L. Beuzit, and D. Mouillet, "Optimisation of Shack-Hartmann based wavefront sensor for XAO system," in Advancements in Adaptive Optics, Proc. SPIE 5490, 1155-1166 (2004).

[11] G. Rousset, F. Lacombe, P. Puget, N. Hubin, E. Gendron, T. Fusco, R. Arsenault, J. Charton, P. Gigan, P. Kern, A.M. Lagrange, P.-Y. Madec, D. Mouillet, D. Rabaud, P. Rabou, E. Stadler and G. Zins, "NAOS, the first AO system of the VLT: on sky performance, " in Adaptive Optical System Technology II, Bellingham, Proc. SPIE 4839, 140$149,(2002)$.

[12] M. Nicolle, T. Fusco, G. Rousset, and V. Michau, "Improvement of Shack-Hartmann wavefront sensor measurement for extreme adaptive optics," Opt. Lett. 29, 2743-2745 (2004).

[13] E. Gendron, "Optimisation de la commande modale en optique adaptative : applications à l'astronomie," Université Paris VII, (1995).

[14] C. Dessenne, "Commande modale et prédictive en optique adaptative classique, "Université Paris VII, (1998).

[15] S. Meimon, , C. Petit, T. Fusco, C. Kulcsár, "An efficient vibration identification algorithm for AO systems," this conférence, paper number

[16] C. Kulcsar, H.-F. Raynaud, C. Petit, and J. -M. Conan, "Can LQG Adaptive Optics Control Cope with Actuator Saturation?," in Adaptive Optics: Analysis and Methods/Computational Optical Sensing and Imaging/Information Photonics/Signal Recovery and Synthesis Topical Meetings on CD-ROM, OSA Technical Digest (CD) (Optical Society of America, 2007), paper PMA1. 